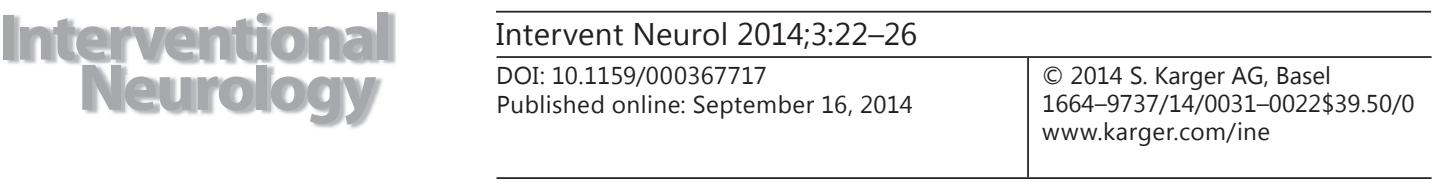

\title{
Stent Placement for Severe Stenosis of the Left Common Carotid Artery with Internal-to-External Carotid Steal
}

\author{
Ryota Kimura $^{a}$ Katsutoshi Takayama ${ }^{b}$ Kaoru Myouchin ${ }^{b} \quad$ Takeshi Wadac $^{c}$ \\ Shinichiro Kurokawa ${ }^{a}$ Kimihiko Kichikawa ${ }^{c}$ \\ Departments of a Neurosurgery and ${ }^{b}$ Radiology and Interventional Neuroradiology, \\ Ishinkai Yao General Hospital, Yao, and ' Department of Radiology, Nara Medical University, \\ Kashihara, Japan
}

\section{Key Words}

Carotid artery stenting $\cdot$ Internal-to-external carotid artery steal · 3 -dimensional time-of-flight magnetic resonance angiography

\begin{abstract}
We report the case of a 64-year-old male with internal carotid artery (ICA)-to-external carotid artery (ECA) steal due to severe stenosis of the common carotid artery (CCA). Left CCA occlusion was initially diagnosed on 3-dimensional time-of-flight magnetic resonance angiography, but digital subtraction angiography revealed severe stenosis of the left CCA and retrograde flow through the left ICA feeding the left ECA. Diverted blood flow from ECA to ICA in cases with occlusion or severe stenosis of the CCA represents a well-known alternative collateral flow pattern called ECA-to-ICA steal. However, collateral flow from ICA to ECA is rarely observed and may be termed ICA-to-ECA steal. We treated CCA stenosis in our patient by carotid artery stenting (CAS) because his CCA stenosis had been gradually progressing since the initial ischaemic attack. Antegrade ICA flow subsequently recovered. To the best of our knowledge, this is the first report of ICA-to-ECA steal normalised by the treatment of CCA stenosis using CAS.

(C) 2014 S. Karger AG, Basel
\end{abstract}

\section{Background}

The internal carotid artery (ICA) is usually collateralised in the presence of occlusion or severe stenosis of the common carotid artery (CCA) by retrograde flow through the external carotid artery (ECA). This process has long been known as carotid steal or ECA-to-ICA 

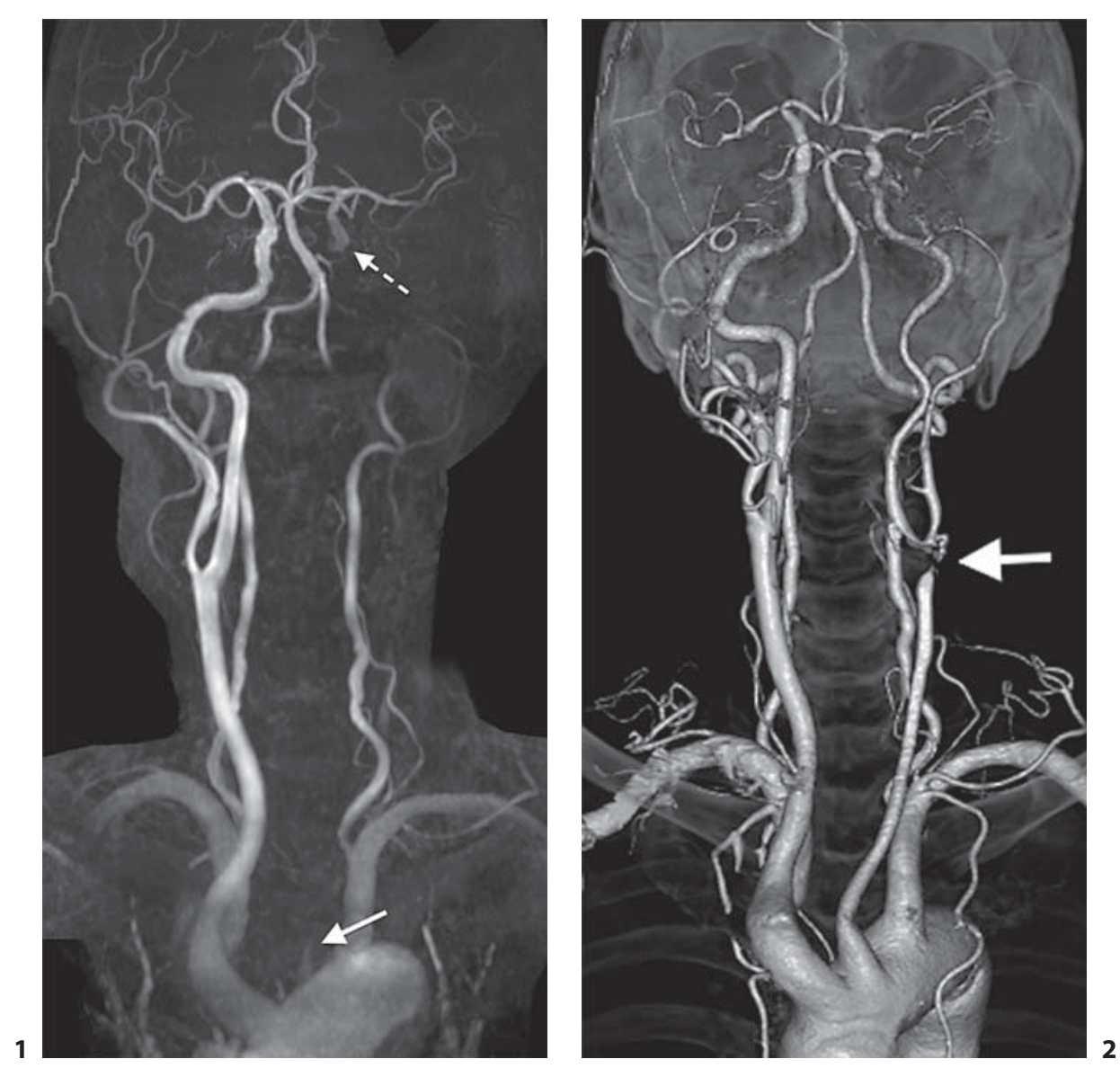

Fig. 1. 3D-TOF MRA. The left CCA seems to be occluded at the proximal part (white arrow). An intradural part of the left ICA is detected (white dotted arrow).

Fig. 2. 3D CTA. Local severe stenosis of the distal part of the left CCA (white arrow) is revealed. The left ICA is patent from the ICA bifurcation.

steal [1]. On the other hand, a collateral flow pattern in which the ECA is fed by retrograde flow through the ICA is rare and could be termed ICA-to-ECA steal. To the best of our knowledge, this is the first reported case of ICA-to-ECA steal in which haemodynamic status was normalised as a result of carotid artery stenting (CAS) for the CCA stenosis.

\section{Case Presentation}

A 64-year-old male had a history of transient motor weakness in the right hand on two occasions. After the initial attack, cervical echography revealed $50 \%$ stenosis of the left ICA origin, and aspirin was prescribed at $100 \mathrm{mg} /$ day. Five years after the second attack, he was sent to our hospital after 3-dimensional time of flight (3D-TOF) magnetic resonance angiography (MRA) (Achieva 1.5-T A-series; Koninklijke Philips Electronics, Amsterdam, The Netherlands) showed occlusion of the left CCA (fig. 1). However, 3D computed tomographic angiography (CTA) using a 64-slice multidetector scanner (Somatom Sensation 64; Siemens AG, Erlangen, Germany) revealed that the left CCA was severely stenotic rather than occluded (fig. 2). Left carotid angiography using a twin flat-panel angiographic system (AXIOM Artis dBA; Siemens AG) revealed severe local stenosis of the left CCA just proximal to the ICA bifurcation. In addition, to-and-fro flow at the ICA origin and slow antegrade flow in the CCA to the ECA were seen (fig. 3a). Left vertebral angiography showed that 

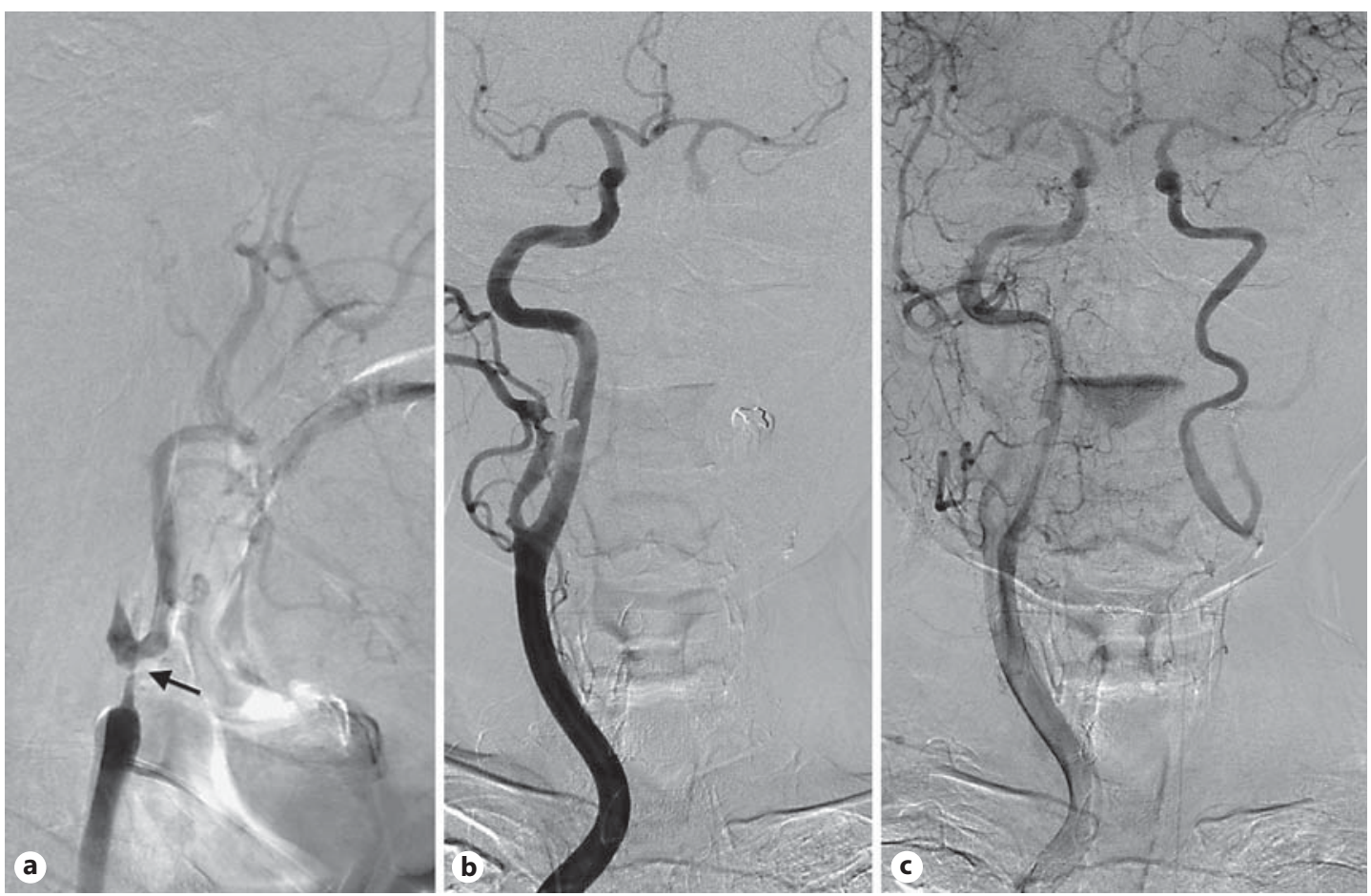

Fig. 3. Digital subtraction angiography (DSA). a Left carotid angiography (lateral view) reveals local severe stenosis of the left CCA (black arrow) along with to-and-fro flow at the ICA origin and slow antegrade flow in the CCA to the ECA. b Right carotid angiography [anteroposterior (AP) view] in the early phase shows a welldeveloped anterior communicating artery and bilateral A1 segment of the ACA allowing good collateral flow to the left ICA territory. $\mathbf{c}$ Right carotid angiography (AP view) in the late phase shows reversed flow of the left ICA reaching the left ICA bifurcation and antegrade flow in the left ECA.

retrograde collateral flow via left vertebral-occipital artery anastomosis only reached the left occipital artery. However, right carotid angiography showed that the anterior communicating artery (AComA) and bilateral A1 segments of the anterior cerebral artery (ACA) were well developed, and right-to-left anterior collateralisation through the AComA led to reversed flow through the left ICA to feed the left ECA (fig. 3b, c).

We performed CAS for the left CCA under local anaesthesia. Cilostazol $(200 \mathrm{mg} /$ day) was additionally prescribed 1 week before the procedure. An 8-F 100-cm guiding catheter (Optimo; Tokai Medical Products, Kasugai, Japan) was inserted into the left CCA via the right femoral approach. Heparin was administered intravenously in 5,000- or 1,000-IU boluses every hour to achieve a periprocedural activated clotting time of $>275$ s. Under distal filter protection (FilterWire EZ; Boston Scientific, Natick, Mass., USA) and proximal balloon protection using the Optimo guiding catheter, pre-dilation was achieved using a balloon catheter of $3.0 \times 40 \mathrm{~mm}$ (Makeway; Cordis, Miami Lakes, Fla., USA) that was then replaced with a self-expanding stent of $8.0 \times 40 \mathrm{~mm}$ (Precise Pro RX; Cordis). The stent was post-dilated using a balloon catheter of $4.0 \times 40 \mathrm{~mm}$ (Aviator; Cordis) under intravenous administration of $0.5 \mathrm{mg}$ atropine (fig. 4a).

The stenosis was successfully treated by CAS (fig. 4b) and left ICA antegrade flow was restored (fig. 4c, d). No neurological deficits developed after the procedure, and the patient was discharged 10 days later.

\section{Discussion}

Subclavian steal is a well-known haemodynamic finding defined as reversed blood flow in the vertebral artery due to proximal stenosis and/or occlusion of the subclavian artery. Contorni [2] was the first to describe it in a patient showing absence of a radial pulse. This 

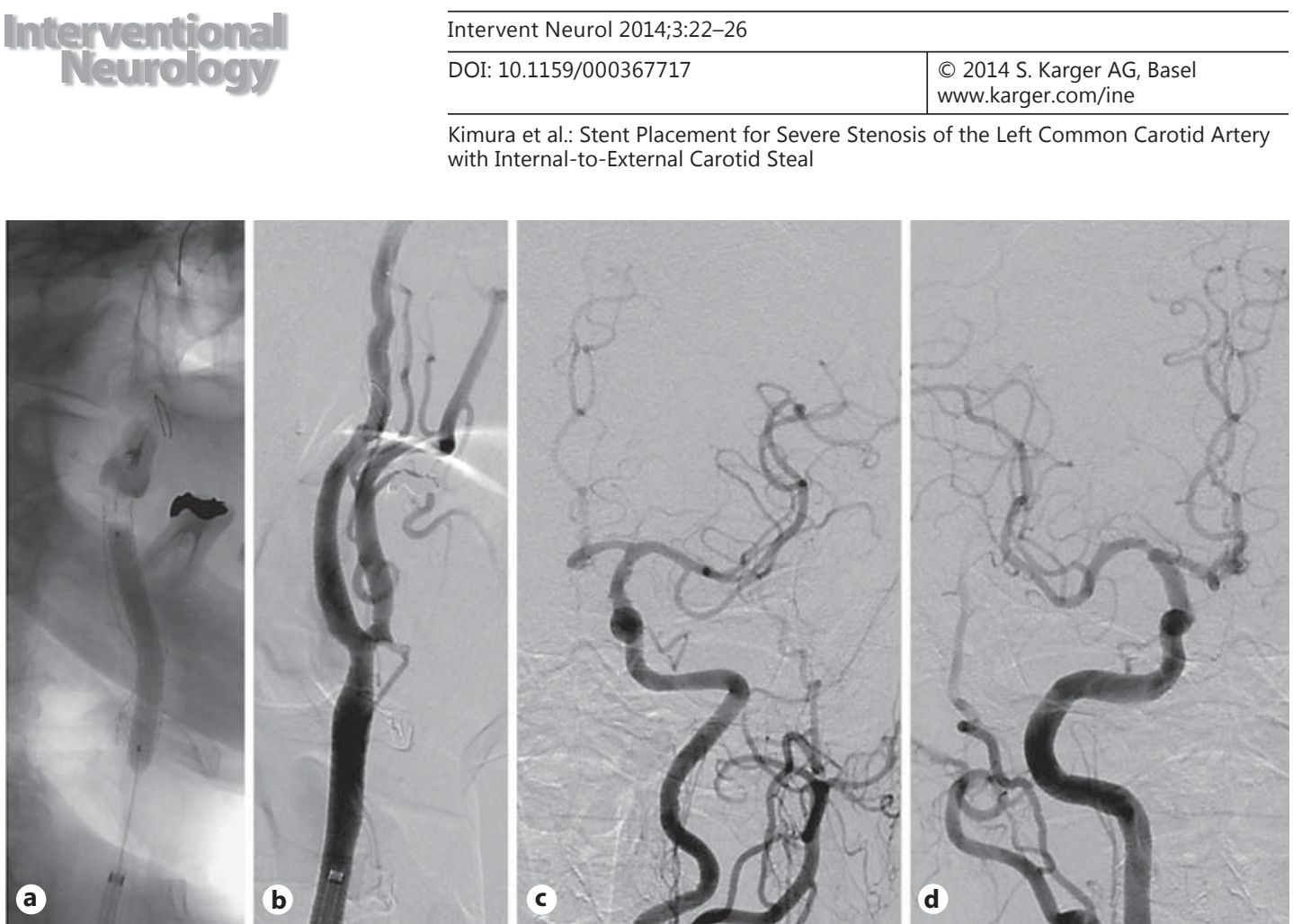

Fig. 4. CAS for stenosis of the left CCA. The stent was placed from the ipsilateral ICA to the CCA. a Live image from left carotid angiography (right anterior oblique view) after dilatation with a balloon (diameter, $4 \mathrm{~mm}$ ) following self-expanding stent placement $(8.0 \times 40 \mathrm{~mm})$ from ICA to CCA. b DSA from left carotid angiography (right anterior oblique view) immediately following dilatation shows successful dilatation of the lesion. c DSA from left carotid angiography (AP view) immediately after CAS shows recovery of antegrade flow in the left ICA. d DSA from right carotid angiography (AP view) immediately after CAS shows disappearance of collateral flow from left ICA territory and retrograde flow to the left ICA.

can on rare occasions lead to subclavian steal syndrome, which causes symptoms such as presyncope, syncope, and a blood pressure differential between the arms. The prevalence of subclavian steal was reported as $5.4 \%$ in a series of 7,881 ultrasound examinations of extracranial neck vessels [3]. In that series, only 38 patients $(8.8 \%$ of cases showing subclavian steal phenomenon) were symptomatic.

Diverted blood flow from ECA to ICA via the carotid bulb is a well-known alternative collateral flow pattern in cases with occlusion of the CCA or innominate artery. Such cases have been reported as carotid steal or ECA-to-ICA steal [1] and have been hypothesised to occur only when cross-circulation via the anterior part of the circle of Willis is inadequate [4]. The prevalence of ECA-to-ICA steal was reported as $0.3 \%$ among 3,463 patients who underwent colour Duplexultrasonography [5]. On the other hand, to the best of our knowledge, ICA-to-ECA steal has been reported in only one case [6], which was detected using a combination of transcranial Doppler and Duplex imaging. The reasons underlying ICA-to-ECA steal in this case are unclear, but we speculate that specific anatomical features may have been involved. First, the AComA and bilateral A1 segments of the ACA were well developed. Second, arteriosclerosis was slight, except for the local CCA stenosis. The presence of adequate collateral blood supply and low vascular resistance from the right ICA via the AComA to the left ICA territory may thus have resulted in ICA-to-ECA steal. In our case, we treated CCA stenosis using CAS for three reasons. First, CCA stenosis had been gradually progressing since the initial ischaemic attack. Second, the frequency of amaurosis fugax has been reported to be significantly higher with CCA stenosis than with ICA stenosis [7]. Finally, left carotid angiography revealed to-and-fro flow at the ICA origin. This might have resulted in cerebral embolism, even if antegrade flow did not reach the intracranial ICA on left carotid angiog- 
raphy. The present case represents the first report of ICA-to-ECA steal in which haemodynamic status was normalised as a result of the treatment of CCA stenosis by CAS.

While 3D-TOF MRA is widely used to evaluate cerebrovascular diseases, the modality has some drawbacks, such as flow void artifacts, loss of signal due to low flow velocity and lack of downstream signals due to pre-saturation [8]. The present case was initially misdiagnosed as CCA occlusion by 3D-TOF MRA. Slow flow in the left CCA seems to have resulted in loss of signal in this vessel, while reversed flow in the left ICA led to a loss of signal due to pre-saturation. If we had not performed a re-evaluation using 3D-CTA, the patient would not have received adequate treatment. Even if a CCA seems to be occluded on 3D-TOF MRA, careful evaluation using other modalities is warranted.

\section{References}

1 Sutton D, Davies ER: Arch aortography and cerebrovascular insufficiency. Clin Radiol 1966;17:330-345.

-2 Contorni L: The vertebro-vertebral collateral circulation in obliteration of the subclavian artery at its origin (in Italian). Minerva Chira 1960;15:268-271.

-3 Labropoulos N, Nandivada P, Bekelis K: Prevalence and impact of the subclavian steal syndrome. Ann Surg 2010;252:166-170.

4 Dashefsky SM, Cooperberg PL, Harrison PB, et al: Total occlusion of the common carotid artery with patent internal carotid artery. Identification with color flow Doppler imaging. J Ultrasound Med 1991;10:417-421.

5 Ozbek SS, Memiş A, Killi R, et al: Carotid steal: report of ten cases. J Ultrasound Med 1998;17:623-629.

6 Kwan KY, Schwend RB, Baker LA, et al: Unusual hemodynamics associated with a common carotid artery occlusion. J Vasc Technol 1993;17:149-152.

7 Hoya K, Morikawa E, Tamura A, et al: Common carotid artery stenosis and amaurosis fugax. J Stroke Cerebrovasc Dis 2008;17:1-4.

-8 Nederkoorn PJ, van der Graaf Y, Eikelboom BC, et al: Time-of-flight MR angiography of carotid artery stenosis: does a flow void represent severe stenosis? AJNR Am J Neuroradiol 2002;23:1779-1784. 